\title{
Attention Modulates Initial Stages of Visual Word Processing
}

\author{
María Ruz and Anna C. Nobre
}

\begin{abstract}
Selective attention has the potential to enhance the initial processing of objects, their spatial locations, or their constituent features. The present study shows that this capacity to modulate initial stages of processing also applies to linguistic attributes. A cueing paradigm focused attention at different levels of word representations on a trial-by-trial basis to study the time course of attentional modulation on visual word processing by means of a high-density electrophysiology recording system. Attention to different linguistic
\end{abstract}

\section{INTRODUCTION}

Language is a highly complex skill of utmost importance to humans. From words to sentences, either in oral or in written language, our brain computes several kinds of representations to be able to communicate ideas. Visual word recognition, a core element in written language, involves a complex series of interactive processes in the brain, which include the analysis of the orthographic, phonological, and semantic attributes of the printed words. Following initial shape extraction common to all visual stimulus categories, neuronal responses occur in a functionally specialized region in the left fusiform gyrus, which responds preferentially to words or letter-string stimuli around 150-200 msec. During this stage, words or word-like stimuli (such as pseudowords or nonwords) are treated differently from other object categories such as nonlinguistic symbols or faces (e.g., Tarkiainen, Cornelissen, \& Salmelin, 2002; Nobre, Allison, \& McCarthy, 1994, 1998). Later, neuronal responses occur in other, specialized brain areas where the meanings, sounds, and contexts of words are computed. Areas showing language-relevant functional specialization include the superior temporal and basal temporal cortex, posterior middle temporal cortex, supramarginal gyrus, and multiple aspects of the inferior frontal gyrus (see Hauk, Davis, Ford, Pulvermuller, \& Marslen-Wilson, 2006; Holcomb \& Grainger, 2006; Salmelin \& Kujala, 2006; Liu \& Perfetti, 2003; Dale et al.,

Oxford University attributes modulated components related to semantic, phonological, and orthographic stages of word processing. Crucially, the N200, associated with initial stages of orthographic decoding, was enhanced by attention to the letter pattern of words. These results suggest that top-down attention has the capacity to enhance initial perceptual stages of visual word processing and support the flexibility of attention in modulating different levels of information processing depending on task goals.
2000). Thus, it seems that the brain initially processes the physical characteristics of the stimuli and then progressively accesses orthographic, phonological, and semantic codes of the words (Hauk et al., 2006; Holcomb \& Grainger, 2006; Liu \& Perfetti, 2003).

Selective attention comprises a variety of mechanisms that can be used to modulate brain activity along several levels of information processing depending on task goals (Luck \& Hillyard, 1999). It has been shown extensively that selective attention can be deployed to spatial locations (Posner, 1980), objects or their features (Anllo-Vento, Luck, \& Hillyard, 1998; Duncan, 1984), motor action (Rushworth, Krams, \& Passingham, 2001), temporal intervals (Coull \& Nobre, 1998), or information held in working memory (Griffin \& Nobre, 2003). One of the most interesting aspects of attentional selection is its temporal profile, as it is informative about the stages of information processing (early vs. late) that are affected by the deployment of attention. Attention to a spatial location enhances target processing as early as $80 \mathrm{msec}$ after stimulus onset, as shown by a modulation of the P1 event-related potential (ERP) component. Attentional selection of objects or features also takes place early in time, starting around 100-150 msec (Schoenfeld et al., 2007; Hillyard \& Anllo-Vento, 1998; Valdes-Sosa, Bobes, Rodriguez, \& Pinilla, 1998). Attention also modulates other, later stages, as indexed by the P300 or the N400 (Vogel, Woodman, \& Luck, 2005). This set of results supports models that postulate that selective attention can bias information processing at many different levels of representation depending on the task at hand (Luck \& Hillyard, 1999). 
Language has often been used as a paradigmatic case of encapsulation from other cognitive functions (Seidenberg, 1985; Fodor, 1983) in the sense that it has its own rules of operation that are not subject to modification by effort and volitionally controlled strategies (Posner \& Carr, 1992). Nowadays, however, this notion has changed. The effects of attention on language have been studied by means of several different paradigms, which seem to converge on the idea that attention has the capacity to affect word processing. Some studies manipulating the allocation of spatial attention, while keeping task demands constant, have shown that attended words generate ERPs with larger P1-N1 and N400 components than do unattended words (McCarthy \& Nobre, 1993). Other studies have manipulated the depth of word processing by means of task demands. For example, it has been shown that the amount of semantic priming, as indexed by the N400 effect (i.e., the difference in amplitude for related vs. unrelated semantic pairs) is significantly reduced when counting words compared to memorizing them (Bentin, Kutas, \& Hillyard, 1993; see also Bentin Kutas, \& Hillyard, 1995; Holcomb, 1988). The attentional blink also affects the brain correlates of word processing (Sergent, Baillet, \& Dehaene, 2005). Other experiments have manipulated the expectations to perform either a verbal or a perceptual/spatial task, to show modulations of language-related ERP components such as the inferior temporal negativity (Lai \& Mangels, 2007) or the N400 (Cristescu \& Nobre, in press; see also Miniussi, Marzi, \& Nobre, 2005). Whereas these and many other studies show that attention modulates language processes, there is also a great deal of reports suggesting that unattended words are also analyzed to a certain extent (e.g., Pesciarelli et al., 2007; Ruz, Wolmetz, Tudela, \& McCandliss, 2005; Ruz, Worden, Tudela, \& McCandliss, 2005; McCann, Remington, \& Van Selst, 2000; Luck, Vogel, \& Shapiro, 1996; Stroop, 1935).

To date, research about attentional effects on languagerelated processing has mostly focused on modulations taking place relatively late, arguably after word recognition has occurred. The N400, thought to reflect the integration of information within a semantic context, seems to be the language-related component most sensitive to attentional manipulations (e.g., Kiefer \& Brendel, 2006; Luck et al., 1996; Bentin et al., 1993; Holcomb, 1988). In some other cases, tasks stressing phonological analyses modulate electrodes at left temporoparietal regions during the so-called N350 component (Bentin, Mouchetant-Rostaing, Giard, Echallier, \& Pernier, 1999). So far, there is no strong evidence for early perceptual modulation within language-specific areas. For example, Nobre et al. (1998) recorded field potentials directly from the inferior surface of the temporal lobe of patients while they attended to either red or green interleaved colored words, and reported that whereas attention had a large impact on potentials over the posterior fusiform gyrus at $400 \mathrm{msec}$, the earliest letter-related responses (150-200 msec) were unaffected. Along the same lines, there is no conclusive evidence of attentional modulations in the N200, an ERP component thought to reflect initial orthographic processes taking place in left fusiform regions. This set of results agrees with a cascade model of visual word recognition in which initial stages related to letter decoding are modulated only by stimulus type, and later ones, which feed from previous ones, are susceptible to attentional top-down modulation by task goals (Bentin et al., 1999).

Attentional selection is characterized by its flexibility. The level of representation at which information is selected depends on the processing stages stressed by the task demands (Nobre, 2004; Luck \& Hillyard, 1999). If the function of early stages of visual word processing is orthographic decoding, and if they are potentially susceptible to attentional influences, their response should be higher under task conditions stressing selection within the orthographic domain, compared to other demands emphasizing phonological or semantic processing. This issue is relevant both to models of attentional selection and to models of language processing. In the first case, proving the potential of selective attention to affect early representations within the language domain would strengthen the case of the ubiquity of attentional selection outside the spatial realm. In the second case, models of visual word processing could benefit from the incorporation of a task-goal factor operating at different levels of linguistic representations depending on the task at hand.

We developed a paradigm in which participants were cued, on a trial-by-trial basis, to pay attention to the orthography, phonology, or semantics of upcoming target words. In the orthographic condition, participants judged of the consonant or vowel nature of letters, which requires access to orthographic codes to map physical features of letters (i.e., shape) into categories of linguistic units (i.e., letters). In the phonological task, participants categorized words as monosyllabic or bisyllabic. In the semantic condition, they judged whether words represented a natural or man-made object. All language tasks used the same set of words displayed under the same perceptual conditions. Participants also performed a nonlinguistic task using similar stimuli and response requirements, in which they judged the shape of letter-like novel symbols. To obtain the temporal course of attentional modulations on word processing, we measured high-density electroencephalographic data from participants during task performance. We expected different task demands to modulate ERP components associated with separate stages of visual word processing. Specifically, we hypothesized that attending to orthography would modulate the amplitude of the N200 measured over left posterior electrodes; focusing on phonological representations would affect the N350 component over left temporoparietal electrodes; and 
semantic analysis would lead to an enhanced N400 in centroparietal regions.

\section{METHODS}

Twenty-four right-handed native English speakers (1927 years, 10 men), with normal or corrected-to-normal vision, were recruited from the University of Oxford community. They all signed a consent form approved by the University of Oxford Research Ethics Committee and received payment in exchange for their participation.

Cues were composed of the letters M, S, L, and the symbol \#, which appeared in a row at the center of the screen. Each character in the cue was colored in red, blue, green, or yellow (see Figure 1). Participants were asked to use the instructions coded by the letter in a specific color in the cue to direct their attention to the task to be performed on the upcoming target. The colors changed from trial to trial. When the $\mathrm{L}$ was presented in the relevant color, they had to perform a task with the letters of the target (decide whether the underlined letter is a consonant or a vowel). The character $\mathrm{S}$ instructed participants to respond to the sound of the word (judge whether the word has one or two syllables). The $\mathrm{M}$ cued them to word meaning (decide whether the word represents something natural or man-made). The \# character prompted the symbols task (decide whether the underlined symbol in the string has a symmetrical or asymmetrical shape). The location of the four characters in the cue $(\mathrm{M}, \mathrm{S}, \mathrm{L}, \#)$ remained constant for a given participant, but their position was counterbalanced across participants. The specific color assigned to each participant was also counterbalanced.

The language tasks (orthographic, phonological, and semantic) used 80 five-letter concrete words as targets, which had an average familiarity of $526(S D=58.6)$ and a mean Kucera frequency of $43.8(S D=66.1)$. A little bar underlined one of the letters in each word. For half of the words, the underlined letter was a consonant, and for the other half, it was a vowel. Forty words were monosyllabic and the other 40 were bisyllabic. Also, half of the words represented a natural item and the other half corresponded to a man-made object. Such orthographic, phonological, and semantic characteristics of the words were orthogonal. To create a set of symbols as similar as possible to words, we rearranged the different parts of individual letters to form 20 symbols with either a symmetrical or an asymmetrical shape (see Figure 1). We then arranged these letter-sized symbols in 40 five-symbol strings, half of which had a symmetrical symbol underlined and the other half an asymmetrical one. All words were used in all language tasks, twice in each. In total, participants saw each word six times and each symbol string four times. No word or symbol was repeated until all items had been presented once. Participants used the index finger in their right or left hand to press one of two buttons on the keyboard to make speeded choice responses for each of the tasks. The response assignment was counterbalanced across participants.

A PC controlled by Presentation 0.70 displayed the stimuli. Each trial comprised the following events (see Figure 1). After a fixation point $\left(+; 0.3^{\circ}\right)$ of variable duration (1500-2500 msec), a cue $\left(1.7^{\circ}\right)$ was flashed in the center of the screen for $200 \mathrm{msec}$. During another variable interval (1300-2300 $\mathrm{msec})$, the fixation point was displayed and then either an uppercase word or a symbol $\left(1.7^{\circ}\right)$ was presented in the same position for 200 msec. Participants were allowed $3000 \mathrm{msec}$ to respond and received auditory feedback (50 msec) after incorrect responses or misses. On average, a trial lasted $7 \mathrm{sec}$. Participants were asked to refrain from blinking
Figure 1. Display of sequence of events during a trial. The cue was briefly presented after a fixation point of variable duration. The color of the characters in the cue, L, S, M, and \#, was either red, blue, green, or yellow and changed unpredictably from trial to trial, instructing the participants which task to perform on the target. After another variable 1500 - to 2500-msec interval, the target was flashed for $200 \mathrm{msec}$. The target was a word in $75 \%$ of the trials and a string of novel symbols in the remaining ones. Participants had 3 sec to respond. The arrow represents the direction of time.

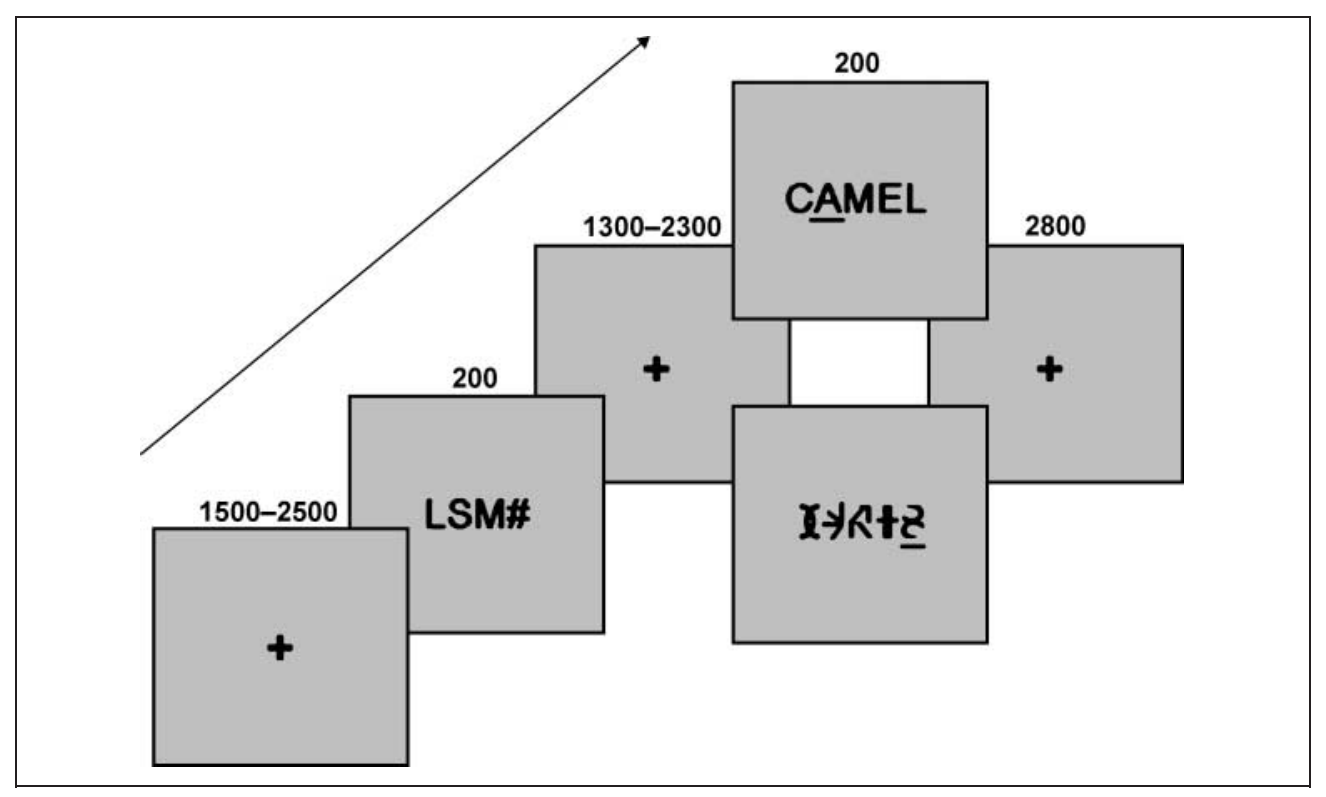


and moving, except during the intervals displaying the fixation point between trials. In total, there were 640 trials (160 per condition), grouped in blocks of 40 trials. Participants were allowed to rest between blocks. Before the main task, participants performed a short training session (10 min) with a different set of words in order to familiarize them with the task. The whole electroencephalogram (EEG) recording session lasted about an hour and a half.

\section{EEG Acquisition and Analyses}

EEG data were collected over 128 electrodes with the Biosemi recording system, which uses active electrodes (Biosemi, Amsterdam, the Netherlands) at a 2048-Hz digitalization rate (417-Hz bandwidth). Data were recorded relative to a common average. Vertical and horizontal oculogram activity was also recorded by means of four electrodes, one above and one below the left eye, and one at each of the outer canthi of the eyes. EEG was transformed off-line with a 30-Hz low-pass filter $(6 \mathrm{~dB} /$ oct), referenced to $\mathrm{Cz}$ and segmented. Epochs encompassed $200 \mathrm{msec}$ before and $800 \mathrm{msec}$ after target presentation. Segments containing blinks (thresholded at $70 \mu \mathrm{V}$ in the eye channels) or other artifacts $(100 \mu \mathrm{V}$ threshold in all channels) were discarded. To explore the effects of attention on target processing, segments were averaged according to the task performed (orthographic, phonological, semantic, or symbol). After averaging, channels containing excessive noise or drift were interpolated using a spline transformation and then data were transformed to an average reference. Due to the low number of error trials and the lack of differences across conditions, both correct and incorrect trials were included in the averages.

In the first place, we were interested in establishing when the processing of words and symbols differed categorically. As an indication, we studied the time points in which the map topographies of the words differed from symbols, which is suggestive of differences in categorical processing between the two types of stimuli. We averaged together all the conditions in which the target was a word and compared the topographies for words versus symbols on a sample-by-sample basis, using a nonparametric topographic analysis of variance (TANOVA; Strik, Fallgatter, Brandeis, \& Pascual-Marqui, 1998) implemented in the Cartool software (brainmapping.unige.ch/ Cartool.php). To perform this analysis, an ERP map is computed for each time point. All the maps are averagereferenced and normalized to the mean paired global field power (GFP); this procedure uses, for every participant, the two paired conditions to calculate the mean GFP across time, and the resulting value is applied to both conditions. To detect systematic differences between the topographies of the two conditions, the maps are randomly shuffled pairwise between the two groups. The mean of the maps of the two new groups is obtained, and then the dissimi- larity value between them is computed. Significant differences indicate that the topographical distribution differs between the two conditions (Lehmann \& Skrandies, 1980). Hence, this TANOVA procedure detects significant variations in topographical maps, which indicate a different pattern or weighting of underlying neural generators across the two conditions. To avoid Type I errors, we only considered significant epochs in which there were more than 40 samples $(20 \mathrm{msec})$ reaching the $p<.05$ criterion.

A complementary analysis tested for the effects of attention on visual word processing. We focused our contrasts on the amplitude of three language-related potentials associated with the orthography (N200), phonology (N350), and semantics (N400) of words. We selected a priori a set of contiguous electrodes in which these components are typically maximally distributed (Bentin et al., 1999). Such regions were left posterior parietal (LPP) for the N200, left temporoparietal (LTP) for the N350, and centromedial (CM) for the N400 (see Figure 4). In addition, to obtain a common set of temporal windows for the analyses of the voltage amplitude of the ERPs, all conditions in which the target was a word were averaged together and submitted to a segmentation analysis performed with Cartool. We used a clustering method to find, at the level of the groupaverage data, the set of topographies that were predominant as a function of time (e.g., Murray et al., 2004). These are obtained by finding periods of stability in the map representation of the normalized ERPs (with the constraint that maps should remain stable for at least $20 \mathrm{msec}$ ) and computing the average map for each of these stable periods. The number of maps that best explained the whole group-averaged data set was defined by a cross-validation criterion (Pascual-Marqui, Michel, \& Lehmann, 1995) by which the minimum number of maps explaining the most part of the variance is chosen.

The amplitude analyses were performed over the time windows reflecting overall periods of stable configuration of generators, which were identified by the segmentation procedure in which the components of interest peaked. These included from 190 to 260 for the N200, from 350 to 390 for the N350, and from 390 to 430 for the N400 component. Then, we calculated the average voltage of all the electrodes in these regions of interest during the selected temporal intervals and introduced these values in a 3 (Temporal Window) $\times 3$ (Region) $\times 3$ (Task) multifactorial measures analysis of variance (ANOVA), followed by planned comparisons. Where appropriate, the degrees of freedom were modified according to the Greenhouse-Geisser correction to compensate for the nonsphericity in the EEG data.

\section{RESULTS}

Tasks were equated in terms of accuracy and reaction time (RT; see Table 1 ). The average accuracy was 0.9 and 
Table 1. Mean Accuracy and Reaction Time per Task

\begin{tabular}{lcccr}
\hline & Orthographic & Phonological & Semantic & Symbols \\
\hline Accuracy & 0.91 & 0.9 & 0.9 & 0.88 \\
SD & 0.06 & 0.06 & 0.05 & 0.07 \\
RT & 1105.6 & 1104.9 & 1117.4 & 1051.2 \\
SD & 285.1 & 284.8 & 252.9 & 278.8 \\
\hline
\end{tabular}

did not differ across tasks, $F(3,21)=2.03, p=.13$. The average RT was $1095 \mathrm{msec}$ and did not differ across tasks, $F(3,21)=1.29, p=.3$. When these comparisons were restricted to the three word conditions, performance measures were still balanced $(F S<1)$.

Results of the TANOVA comparing successive topographies of the ERP elicited by words and symbols are displayed in Figure 2. Differences were significant between 108 and 145 msec (74 consecutive samples, $p<$ .05 ) and between 160 and $708 \mathrm{msec}$ (1095 consecutive samples, $p<.05$ ). In the last segment (up until $800 \mathrm{msec}$ ), samples had interleaved values over and below significance level (see Figure 2).

The complementary segmentation analyses yielded nine different maps explaining $95.5 \%$ of the variance in the ERPs associated to word processing, starting at $95 \mathrm{msec}$ (displayed in Figure 3). The first map corresponded to the P1 component, and consisted of positive voltages at lateral posterior electrodes and negative values at frontal regions. By $125 \mathrm{msec}$, the polarity reversed at posterior locations, which remained negative until 260 msec. Initially, the negative potentials were symmetrically distributed over lateral posterior electrodes (N1 potential), but they became highly left lateralized between 190 and 260 msec (N200). In the following map, the N300 was visible in left frontal regions. The final topographies of the segmentation display consisted of negative and positive voltages, probably reflecting the overlapping N400 and P300 (Bentin, McCarthy, \& Wood, 1985).

Results of voltage comparisons and representative electrodes of the three regions of interest are displayed in Figure 4. Repeated measures ANOVA with the factors Temporal Window (3), Region (3), and Language Task (3) showed a three-way interaction between the factors, $F(4.31,99.13)=5.4, p<.001$. During the time window of the N200 (190-260 msec), ${ }^{1}$ there was an interaction between region and task, $F(1.97,45.31)=3.52, p<.05$. In the LPP region, where the N200 was most pronounced, the ERP of the orthographic task was more negative than for the phonological, $F(1,23)=15.22$, $p<.001$, and semantic tasks, $F(1,23)=11.21, p<.01$. Surprisingly, in the LTP region, the ERP of the phonological task was more negative than that of the semantic condition, $F(1,23)=6.58, p=.01$. No other effects were significant during this temporal window.
We ran an additional analysis to explore an alternative explanation to the early effect centered on the orthographic effect in the LPP area. It could be argued that this effect was merely due to the need of target selection within the word, which was required for the orthographic but not for the phonological or semantic tasks. We compared the amplitude of the N200 for the orthography and symbols condition (which shared the need for target selection within a string of characters). The voltage was more negative for the orthographic task than for the symbols, $F(1,23)=41.81, p<.001$.

During the time window of the N350 (350-390 msec), the interaction between region and task was significant, $F(2.36,54.27)=6.02, p=.003$. As predicted, in the LTP region the ERP of the phonological task was more negative than that of the orthographic, $F(1,23)=7.26$, $p<.01$, and the semantic conditions, $F(1,23)=38.88$, $p<.001$. In the LPP region, the ERP of the phonological task was more negative than the ERP of the orthographic condition, $F(1,23)=5.88, p<.05$. In the CM region, the voltage of the semantic task was more negative than that of the orthographic condition, $F(1,23)=5.47, p=.02$. No other effects were significant during this temporal window.

One of the characteristics of the N200 and N350 components is their lateralization to electrodes in left regions of the scalp. We could not include laterality as an initial factor in the omnibus ANOVA because the electrodes corresponding to the N400 were located in the center of the scalp. Instead, we explored the lateralization of the two components in a separate analysis. To do this, we introduced the average values of the electrodes in the left posterior parietal (N200) and left temporoparietal (N350) regions, together with the average values of symmetrical regions of the scalp, in an ANOVA with the factors Hemisphere (left vs. right), Temporal Window

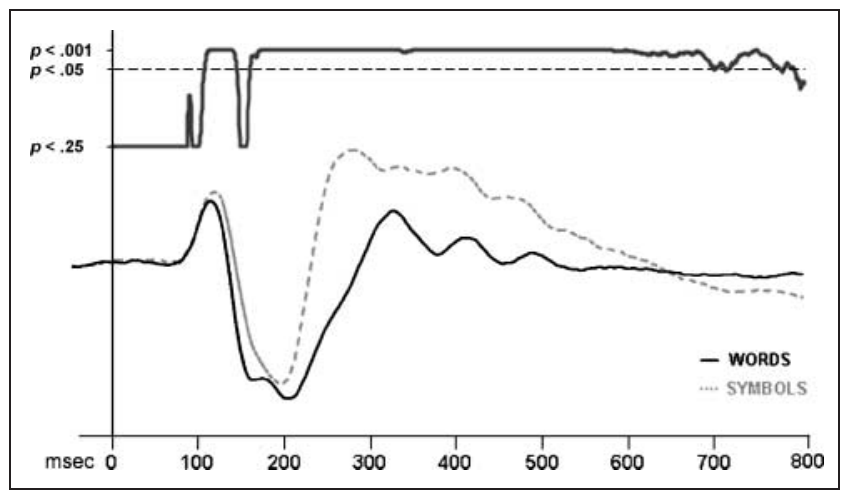

Figure 2. The top part of the figure represents the TANOVA results comparing the dissimilarity between the topographical distribution of all word targets averaged together and the symbols. Differences were significant from 108 to $145 \mathrm{msec}$ and from $160 \mathrm{msec}$ until the latest part of the epoch (see Results), indicating dissociable neural substrates for words and symbols during these time windows. The bottom of the figure displays a representative electrode at left posterior regions (TP7) showing the ERPs for the two categories. 


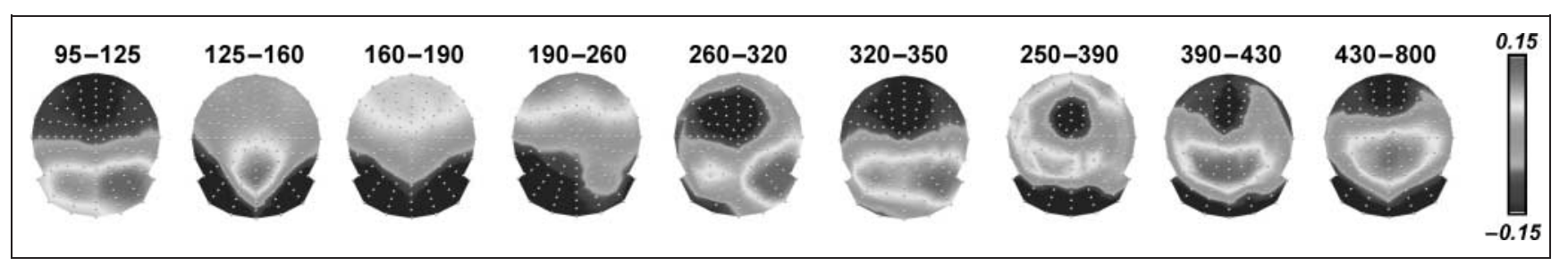

Figure 3. The segmentation analysis of all words averaged together resulted in nine maps, from 95 to $800 \mathrm{msec}$, explaining $95.5 \%$ of the variance in the ERP data. The first map, from 95 to $125 \mathrm{msec}$, represents the temporal window of the P1 component. At 125 msec, the polarity reverses and becomes negative in posterior electrodes and positive in centromedial ones. By $190 \mathrm{msec}$, the posterior negativity is left lateralized, consistent with the topographical distribution of the N200 component, associated with letter-decoding processes. At 260, anterior and inferior posterior electrodes become negative, and a positivity dominates centroposterior channels. This distribution continues, across different topographies, until the end of the epoch.

(200 vs. $350 \mathrm{msec}$ ), Location (LPP vs. LTP), and Task (orthographic, phonological, and semantic). The relevant three-way interaction between Hemisphere, Location, and Task was significant, $F(1.42,32.82)=11.57, p=.001$, in accordance with the left lateralization of the task effects on the N200 and N350 components.

Finally, during the time window of the N400 (390$430 \mathrm{msec}$ ), the interaction between region and task was significant once again, $F(2.9,66.9)=12.90, p<.001$. As predicted, in the CM region the voltage of the semantic task was more negative than either the orthographic, $F(1,23)=4.85, p<.05$, or the phonological, $F(1,23)=$ $8.72, p=.007$, conditions. This effect was reversed in the LTP region, in which the ERP of the semantic task was more positive than those of the orthographic, $F(1,23)=6.33, p<.05$, and phonological, $F(1,23)=$ $37.24, p<.001$, tasks. No other effects were significant during this temporal window.

\section{DISCUSSION}

The goal of our study was to explore the susceptibility of different stages of visual word processing to task settings focusing attention on different levels of analysis within the language domain. Our results show that attention to orthography enhances the word-related N200 component in electrodes over the left posterior parietal scalp, which reflects orthographic analyses. Another early but unexpected effect took place around $200 \mathrm{msec}$ in left parietotemporal electrodes: In this region the peak of the N200 was enhanced for tasks stressing phonological word processing, compared to semantics. In line with our predictions and in agreement with previous literature, attention to phonology enhanced the N350 at left temporoparietal locations, whereas attention to semantics enhanced the N400 in centromedial electrodes. The modulation of languagerelated components by task sets suggests that different levels of language processing can be modulated by endogenous attention. Most importantly, it suggests that even the initial stages of visual word processing are susceptible to attentional enhancement. This lends further support to a characterization of attention as oper- ating through highly flexible mechanisms that can be applied over initial linguistic representations.

Different stages of word processing in our experiment were reflected in a series of maps, corresponding to dissociable functional neural states (Figure 3). Among these, we focused our analyses in the temporal windows encompassing three components known to be associated with orthographic, phonological, and semantic analyses of words. Most previous reports had suggested the effects of attention on language are limited to processing taking place late in time, around $400 \mathrm{msec}$, centered in the N400 component (Nobre et al., 1998; Bentin et al., 1993, 1995; Holcomb, 1988), or in the $300 \mathrm{msec}$ range, when tasks stressed phonological processing (Proverbio, Vecchi, \& Zani, 2004; Bentin et al., 1999). Earlier stages related to orthographic processing had mostly been considered to take place in a common manner, irrespective of task demands (Maurer, Brandeis, \& McCandliss, 2005; Bentin et al., 1999; Allison, McCarthy, Nobre, Puce, \& Belger, 1994). Our results show, in agreement with previous literature, that a task directing attention to the semantic representation of words enhances the negativity associated with the N400 component in centromedial regions (Lai \& Mangels, 2007; Miniussi et al., 2005; Holcomb, 1988). Along the same lines, a task stressing phonological processing enhances the N350 component, registered over left temporoparietal regions (Bentin et al., 1999). As a whole, the differential modulation of attention to phonology and semantics is also in agreement with previous neuroimaging literature showing differential enhanced brain activations for phonological and semantic tasks ${ }^{2}$ (e.g., Snyder, Feigenson, \& Thompson-Schill, 2007; Gitelman, Nobre, Sonty, Parrish, \& Mesulam, 2005). Attention to phonology also modulated the same set of electrodes at an earlier window, from 190 to $260 \mathrm{msec}$. Although unexpected, the localization of this effect in a set of electrodes that typically display phonological modulations suggests that the expectations created by a cue focusing attention on the phonology of words are capable of enhancing brain processing from an early point in time. The data suggest a parallel of this anticipation in the $\mathrm{N} 400$, as the voltage of the semantic task is already more negative than the 


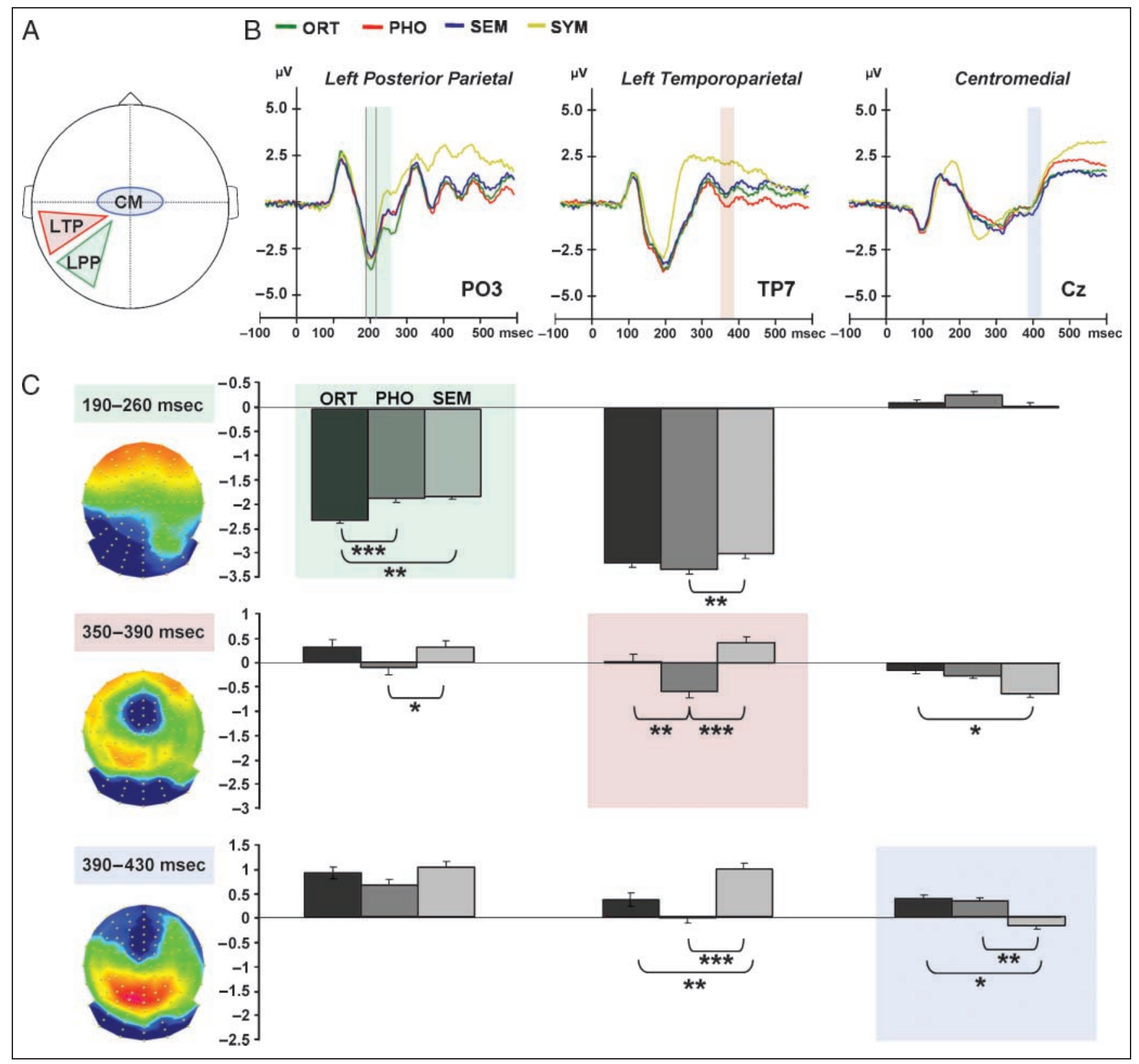

Figure 4. (A) Distribution of the three regions used for the analyses: LPP $=$ left posterior parietal; LTP $=$ left temporoparietal; CM $=$ centromedial. (B) Representative channels of the three regions displaying the ERPs for the three word tasks (Ort = orthography; Pho $=$ phonology; Sem $=$ semantic) and symbols (Sym). (C) Bar graphs displaying the effect of the word task on the voltage averaged across the three regions of interest during the three temporal windows of the N200 (190-260), N350 (350-390), and N400 (390-430) components, together with the topographical maps associated to those epochs.

orthographic task from 350 to $390 \mathrm{msec}$, which foreshadows what will happen in the successive interval over the relevant electrodes.

When attention was directed at the letters embedded in the words, the N200 component was enhanced in electrodes at posterior parietal locations. In our experiment, the same words were used across all tasks, and they were all presented under the same perceptual conditions on a dynamical trial-by-trial basis. The N200 is thought to reflect the initial orthographic processing that takes place in left posterior fusiform regions
(McCandliss, Cohen, \& Dehaene, 2003; Dehaene et al., 2001), a connection that is strengthened by reports of correlation between the amplitude of this component and extent of activation in this fusiform region, as measured by functional magnetic resonance imaging (Brem et al., 2006). Note that at this point in time, the TANOVA analysis comparing the dissimilarity between word and symbol topographies (see Figure 2) suggests that words and symbols are treated as separate categories (Tarkiainen, Helenius, Hansen, Cornelissen, \& Salmelin, 1999). All this taken together lends support to the idea that brain activity 
in left fusiform regions, related to orthographic analyses, is enhanced from an early point in time when task demands focus attention on letters within words.

Recently, there has been another report of early modulations of the N150 component (Spironelli \& Angrilli, 2007). The authors compared the ERPs generated by words in a case-matching task with the ERPs that words generated in a rhyming and a semantic task, presented in different blocks. The amplitude of the N150 component, peaking at $130 \mathrm{msec}$, was enhanced in the case-matching task relative to the two other conditions. Such an early ERP effect, however, most likely was affected by the perceptual differences of the stimuli used across tasks. Whereas the case-matching task used both uppercase and lowercase words, only the latter presentation type was used in the other two conditions. ${ }^{3}$ Differences in behavioral performance across tasks may have also influenced the ERP amplitudes. In addition, the authors did not include a nonlinguistic control condition in the design. This makes it difficult to interpret whether their N150 corresponds to the language-related N200 negativity or to general visual-perceptual processing indexed by the N1. This is a relevant control, as modulations in early components not related to linguistic processing (P1-N1) have also been observed for words presented either within or outside the focus of spatial attention (McCarthy \& Nobre, 1993). In our experiment, the topographical analysis suggests that the N200 occurs at a point in time in which the brain is already treating words and symbols as separate categories, which lends support to the notion that a task focusing attention on letters enhances the orthographic processing indexed by the N200. In our case, however, we cannot rule out completely the alternative explanation that such enhancement was merely due to the need for target selection among distracters. Although the ANOVA comparing words and symbols (which share the same selection demands) showed significant differences, these results may be influenced by the different amplitudes that characterize the N200 component of these two types of stimuli. Future experiments testing local versus global analyses of words versus symbols would be useful.

There have also been many reports of early effects in visual word processing driven by stimulus attributes or priming. Some variables associated to early modulations are lexical features (Sereno, Rayner, \& Posner, 1998; Dehaene, 1995), word frequency (Sereno, Brewer, \& O'Donnell, 2003; Assadollahi \& Pulvermuller, 2001), or even semantic factors (Pulvermuller, Assadollahi, \& Elbert, 2001). Along the same lines, repetition, phonological, and semantic priming effects have been reported during similar time windows (Holcomb \& Grainger, 2006; Proverbio et al., 2004; Ruz, Madrid, Lupiáñez, \& Tudela, 2003; Abdullaev \& Posner, 1998). These effects of stimulus attributes suggest that the language system in the brain is tuned to extract some word-related characteristics at a very fast pace. Priming demonstrations, on the other hand, suggest that the pattern of activation generated by a stimulus leaves a trace that may affect the subsequent processing of another related stimulus early in time.

In our experiment, all effects of stimulus features, priming, and difficulty were equated across tasks. Our results suggest a selective enhancement of specific linguistic processes when top-down attention is focused on different language tasks. Such attentional effects on word processing could be generated in part by the specific preparatory effects initiated by the cues on a trial-by-trial basis. This notion receives support from the pattern of dissociable brain activity that is generated by the cues in this paradigm (Ruz \& Nobre, 2008). We analyzed the topographical maps associated to the different language cues before target presentation. These results showed that, whereas brain activity was indistinguishable across tasks at the beginning of the interval, the topographical maps differed at the end of the epoch by the time target onset approached. During this time window, each task cue generated distinct topographical activity corresponding to dissociable neural generators of preparatory activity for each task condition. This suggests that the generators of anticipatory-biasing brain states for different language goals vary depending on the nature of the task, which in turn may affect the way targets are processed from an early point in time.

In conclusion, the results presented in this article suggest that the informational content of cues focusing attention on different types of computation and the corresponding relevant representation within the language system generates top-down attentional expectations, which modulate various stages of word processing differentially. This finding draws an analogy between mechanisms of selection within space with mechanisms of selection of abstract representations within the language domain and lends further support to the flexibility of attentional selection according to task goals.

\section{Acknowledgments}

This research was supported by a grant from the Biotechnology and Biological Sciences Research Council (BBSRC), UK, to A. C. N. and by an Astor Junior Research Fellowship, New College (Oxford, UK) to M. R.

The Cartool software (brainmapping.unige.ch/Cartool.php) has been programmed by Denis Brunet from the Functional Brain Mapping Laboratory, Geneva, Switzerland, and is supported by the Center for Biomedical Imaging (CIBM) of Geneva and Lausanne.

Reprint requests should be sent to María Ruz, Department of Experimental Psychology, South Parks Road, Oxford OX1 3UD, United Kingdom, or via e-mail: maria.ruz@psy.ox.ac.uk.

\section{Notes}

1. In other previous studies, the $\mathrm{N} 200$ has been reported to peak earlier in time (i.e., around $170 \mathrm{msec}$, e.g., Bentin et al., 1999). In our experiment, this component peaked at $200 \mathrm{msec}$, 
which corresponds to the temporal window we chose for our analyses. When we analyzed the previous temporal interval (160-190 msec) to look for possible task effects, none were significant (all $F \mathrm{~S}<1.3$ ).

2. Many neuroimaging reports show semantic and phonological task-related activations in the left inferior frontal gyrus. However, we opted for not considering left frontal electrodes given that we did not have any a priori prediction regarding task modulations in this region of the scalp. In addition, visual analysis of our data hinted that modulations in this set of frontal electrodes may have taken place later in time (around $400 \mathrm{msec}$ ) and only differentiated between the orthographic and the other two tasks.

3. Spironelli and Angrilli (2007) report an ANOVA that they claim shows that the amplitude of the N150 is not affected by word case. They report a three-way Case $\times$ Region $\times$ Laterality interaction, which failed to reach significance. This shows that the case of the words does not change the pattern of left lateralization of the component (a result that makes sense, given that the stimuli are words in both case conditions). However, this lack of interaction does not prove that the word case changed the amplitude of the component. This could have been shown by comparing the amplitude of the N150 for lower and uppercase words and showing no differences. This contrast was not reported though.

\section{REFERENCES}

Abdullaev, Y. G., \& Posner, M. I. (1998). Event-related brain potential imaging of semantic encoding during processing single words. Neuroimage, 7, 1-13.

Allison, T., McCarthy, G., Nobre, A., Puce, A., \& Belger, A. (1994). Human extrastriate visual cortex and the perception of faces, words, numbers, and colors. Cerebral Cortex, 4 , 544-554.

Anllo-Vento, L., Luck, S. J., \& Hillyard, S. A. (1998). Spatio-temporal dynamics of attention to color: Evidence from human electrophysiology. Human Brain Mapping, 6, 216-238.

Assadollahi, R., \& Pulvermuller, F. (2001). Neuromagnetic evidence for early access to cognitive representations. NeuroReport, 12, 207-213.

Bentin, S., Kutas, M., \& Hillyard, S. A. (1993). Electrophysiological evidence for task effects on semantic priming in auditory word processing. Psychophysiology, 30, 161-169.

Bentin, S., Kutas, M., \& Hillyard, S. A. (1995). Semantic processing and memory for attended and unattended words in dichotic listening: Behavioral and electrophysiological evidence. Journal of Experimental Psychology. Human Perception and Performance, 21, 54-67.

Bentin, S., McCarthy, G., \& Wood, C. C. (1985). Event-related potentials, lexical decision and semantic priming. Electroencephalography and Clinical Neurophysiology, 60, 343-355.

Bentin, S., Mouchetant-Rostaing, Y., Giard, M. H., Echallier, J. F., \& Pernier, J. (1999). ERP manifestations of processing printed words at different psycholinguistic levels: Time course and scalp distribution. Journal of Cognitive Neuroscience, 11, 235-260.

Brem, S., Bucher, K., Halder, P., Summers, P., Dietrich, T., Martin, E., et al. (2006). Evidence for developmental changes in the visual word processing network beyond adolescence. Neuroimage, 29, 822-837.

Coull, J. T., \& Nobre, A. C. (1998). Where and when to pay attention: The neural systems for directing attention to spatial locations and to time intervals as revealed by both PET and fMRI. Journal of Neuroscience, 18, 7426-7435.

Cristescu, T. C., \& Nobre, A. C. (in press). Differential modulation of word recognition by semantic and spatial orienting of attention. Journal of Cognitive Neuroscience.

Dale, A. M., Liu, A. K., Fischl, B. R., Buckner, R. L., Belliveau, J. W., Lewine, J. D., et al. (2000). Dynamic statistical parametric mapping: Combining fMRI and MEG for high-resolution imaging of cortical activity. Neuron, 26, 55-67.

Dehaene, S. (1995). Electrophysiological evidence for category-specific word processing in the normal human brain. NeuroReport, 6, 2153-2157.

Dehaene, S., Naccache, L., Cohen, L., Bihan, D. L., Mangin, J. F., Poline, J. B., et al. (2001). Cerebral mechanisms of word masking and unconscious repetition priming. Nature Neuroscience, 4, 752-758.

Duncan, J. (1984). Selective attention and the organization of visual information. Journal of Experimental Psychology. General, 113, 501-517.

Fodor, J. A. (1983). Modularity of mind: An essay on faculty psychology. Cambridge: MIT Press.

Gitelman, D. R., Nobre, A. C., Sonty, S., Parrish, T. B., \& Mesulam, M. M. (2005). Language network specializations: An analysis with parallel task designs and functional magnetic resonance imaging. Neuroimage, 26, 975-985.

Griffin, I. C., \& Nobre, A. C. (2003). Orienting attention to locations in internal representations. Journal of Cognitive Neuroscience, 15, 1176-1194.

Hauk, O., Davis, M. H., Ford, M., Pulvermuller, F., \& Marslen-Wilson, W. D. (2006). The time course of visual word recognition as revealed by linear regression analysis of ERP data. Neuroimage, 30, 1383-1400.

Hillyard, S. A., \& Anllo-Vento, L. (1998). Event-related brain potentials in the study of visual selective attention. Proceedings of the National Academy of Sciences, U.S.A., 95, 781-787.

Holcomb, P. J. (1988). Automatic and attentional processing: An event-related brain potential analysis of semantic priming. Brain and Language, 35, 66-85.

Holcomb, P. J., \& Grainger, J. (2006). On the time course of visual word recognition: An event-related potential investigation using masked repetition priming. Journal of Cognitive Neuroscience, 18, 1631-1643.

Kiefer, M., \& Brendel, D. (2006). Attentional modulation of unconscious "automatic" processes: Evidence from event-related potentials in a masked priming paradigm. Journal of Cognitive Neuroscience, 18, 184-198.

Lai, G., \& Mangels, J. A. (2007). Cueing effects on semantic and perceptual categorization: ERPs reveal differential effects of validity as a function of processing stage. Neuropsychologia, 45, 2038-2050.

Lehmann, D., \& Skrandies, W. (1980). Reference-free identification of components of checkerboard-evoked multichannel potential fields. Electroencephalography and Clinical Neurophysiology, 48, 609-621.

Liu, Y., \& Perfetti, C. A. (2003). The time course of brain activity in reading English and Chinese: An ERP study of Chinese bilinguals. Human Brain Mapping, 18, 167-175.

Luck, S. J., \& Hillyard, S. A. (1999). The operation of selective attention at multiple stages of processing: Evidence from monkey and human electrophysiology. In M. S. Gazzaniga (Ed.), The new cognitive neurosciences (pp. 687-700). Cambridge: MIT Press.

Luck, S. J., Vogel, E. K., \& Shapiro, K. L. (1996). Word meanings can be accessed but not reported during the attentional blink. Nature, 383, 616-618.

Maurer, U., Brandeis, D., \& McCandliss, B. D. (2005). Fast, visual specialization for reading in English revealed by the 
topography of the N170 ERP response. Behavioral and Brain Functions, 1, 13.

McCandliss, B. D., Cohen, L., \& Dehaene, S. (2003). The visual word form area: Expertise for reading in the fusiform gyrus. Trends in Cognitive Sciences, 7, 293-299.

McCann, R. S., Remington, R. W., \& Van Selst, M. (2000). A dual-task investigation of automaticity in visual word processing. Journal of Experimental Psychology. Human Perception and Performance, 26, 1352-1370.

McCarthy, G., \& Nobre, A. C. (1993). Modulation of semantic processing by spatial selective attention. Electroencephalography and Clinical Neurophysiology, 88, 210-219.

Miniussi, C., Marzi, C. A., \& Nobre, A. C. (2005). Modulation of brain activity by selective task sets observed using event-related potentials. Neuropsychologia, 43, 1514-1528.

Murray, M. M., Michel, C. M., Grave de Peralta, R., Ortigue, S., Brunet, D., Gonzalez Andino, S., et al. (2004). Rapid discrimination of visual and multisensory memories revealed by electrical neuroimaging. Neuroimage, 21, 125-135.

Nobre, A. C. (2004). Probing the flexibility of attentional orienting in the human brain. In M. I. Posner (Ed.), Cognitive neuroscience of attention (pp. 157-179). New York: Guilford Press.

Nobre, A. C., Allison, T., \& McCarthy, G. (1994). Word recognition in the human inferior temporal lobe. Nature, 372, 260-263.

Nobre, A. C., Allison, T., \& McCarthy, G. (1998). Modulation of human extrastriate visual processing by selective attention to colours and words. Brain, 121, 1357-1368.

Pascual-Marqui, R. D., Michel, C. M., \& Lehmann, D. (1995). Segmentation of brain electrical activity into microstates: Model estimation and validation. IEEE Transactions on Biomedical Engineering, 42, 658-665.

Pesciarelli, F., Kutas, M., Dell'acqua, R., Peressotti, F., Job, R., \& Urbach, T. P. (2007). Semantic and repetition priming within the attentional blink: An event-related brain potential (ERP) investigation study. Biological Psychology, 76, 21-30.

Posner, M. I. (1980). Orienting of attention. The Quarterly Journal of Experimental Psychology, 32, 3-25.

Posner, M. I., \& Carr, T. H. (1992). Lexical access and the brain: Anatomical constraints on cognitive models of word recognition. American Journal of Psychology, 105, 1-26.

Proverbio, A. M., Vecchi, L., \& Zani, A. (2004). From orthography to phonetics: ERP measures of grapheme-to-phoneme conversion mechanisms in reading. Journal of Cognitive Neuroscience, 16, 301-317.

Pulvermuller, F., Assadollahi, R., \& Elbert, T. (2001). Neuromagnetic evidence for early semantic access in word recognition. European Journal of Neuroscience, 13, 201-205.

Rushworth, M. F., Krams, M., \& Passingham, R. E. (2001). The attentional role of the left parietal cortex: The distinct lateralization and localization of motor attention in the human brain. Journal of Cognitive Neuroscience, 13, 698-710.

Ruz, M., Madrid, E., Lupiáñez, J., \& Tudela, P. (2003). High density ERP indices of conscious and unconscious semantic priming. Brain Research. Cognitive Brain Research, 17, 719-731.

Ruz, M., \& Nobre, A. C. (2008). Dissociable top-down anticipatory neural states for different linguistic dimensions. Neuropsychologia, 46, 1151-1160.
Ruz, M., Wolmetz, M. E., Tudela, P., \& McCandliss, B. D. (2005). Two brain pathways for attended and ignored words. Neuroimage, 27, 852-861.

Ruz, M., Worden, M. S., Tudela, P., \& McCandliss, B. D. (2005). Inattentional amnesia to words in a high attentional load task. Journal of Cognitive Neuroscience, 17, 768-776.

Salmelin, R., \& Kujala, J. (2006). Neural representation of language: Activation versus long-range connectivity. Trends in Cognitive Sciences, 10, 519-525.

Schoenfeld, M., Hopf, J. M., Martinez, A., Mai, H., Sattler, C., Gasde, A., et al. (2007). Spatio-temporal analysis of feature-based attention. Cerebral Cortex, 17, 2468-2477.

Seidenberg, M. S. (1985). The time course of information activation and utilization in visual word recognition. In D. Besner, T. G. Walker, \& G. E. Mackinnon (Eds.), Reading research: Advances in theory and practice (vol. 5, pp. 200-252). Orlando, FL: Academic Press.

Sereno, S. C., Brewer, C. C., \& O’Donnell, P. J. (2003). Context effects in word recognition: Evidence for early interactive processing. Psychological Science, 14, 328-333.

Sereno, S. C., Rayner, K., \& Posner, M. I. (1998). Establishing a time-line of word recognition: Evidence from eye movements and event-related potentials. NeuroReport, 9, $2195-2200$.

Sergent, C., Baillet, S., \& Dehaene, S. (2005). Timing of the brain events underlying access to consciousness during the attentional blink. Nature Neuroscience, 8, 1391-1400.

Snyder, H. R., Feigenson, K., \& Thompson-Schill, S. L. (2007). Prefrontal cortical response to conflict during semantic and phonological tasks. Journal of Cognitive Neuroscience, 19, 761-775.

Spironelli, C., \& Angrilli, A. (2007). Influence of phonological, semantic and orthographic tasks on the early linguistic components N150 and N350. International Journal of Psychophysiology, 64, 190-198.

Strik, W. K., Fallgatter, A. J., Brandeis, D., \& Pascual-Marqui, R. D. (1998). Three-dimensional tomography of event-related potentials during response inhibition: Evidence for phasic frontal lobe activation.

Electroencephalography and Clinical Neurophysiology, 108, 406-413.

Stroop, J. R. (1935). Studies of interference in serial verbal reactions. Journal of Experimental Psychology, 18, 643-662.

Tarkiainen, A., Cornelissen, P. L., \& Salmelin, R. (2002). Dynamics of visual feature analysis and object-level processing in face versus letter-string perception. Brain, 125, 1125-1136.

Tarkiainen, A., Helenius, P., Hansen, P. C., Cornelissen, P. L., \& Salmelin, R. (1999). Dynamics of letter string perception in the human occipitotemporal cortex. Brain, 122, 2119-2132.

Thierry, G., Martin, C. D., Downing, P., \& Pegna, A. J. (2007). Controlling for interstimulus perceptual variance abolishes N170 face selectivity. Nature Neuroscience, 10, 505-511.

Valdes-Sosa, M., Bobes, M. A., Rodriguez, V., \& Pinilla, T. (1998). Switching attention without shifting the spotlight object-based attentional modulation of brain potentials. Journal of Cognitive Neuroscience, 10, 137-151.

Vogel, E. K., Woodman, G. F., \& Luck, S. J. (2005). Pushing around the locus of selection: Evidence for the flexible-selection hypothesis. Journal of Cognitive Neuroscience, 17, 1907-1922. 